\title{
(14) \\ A Local Search Algorithm to Find a Scheduling Table for Real-Time Systems*
}

\author{
Hideki HASHIMOTO**, Taichiro SUGIYAMA***, Yuma TANAKA** and \\ Mutsunori YAGIURA** \\ ** Graduate School of Information Science, Nagoya University \\ Furocho, Chikusaku, Nagoya 464-8603, Japan \\ E-mail: hasimoto@nagoya-u.jp \\ *** NTT Communications Corporation \\ 1-1-6 Uchisaiwai-cho, Chiyoda-ku, Tokyo 100-8019, Japan
}

\begin{abstract}
A real-time system receives input from the outside, processes it through a sequence of tasks and outputs the result to the outside. Each process corresponds to a sequence of tasks called path and the time from input to output is called response time. For each path, a response time must always be within a specified time. Our objective is to schedule tasks so that the time constraints for all paths are satisfied. In this paper, we adopt a scheduling method based on a scheduling table, which specifies a schedule of a finite time period and is used repeatedly to define the schedule of longer time periods. In order to calculate response times for a schedule efficiently, we introduce a concept called active path. In our algorithm, a scheduling table is searched by a local search. The neighborhood search is accelerated by using precedence relations that are introduced to preserve active paths of a scheduling table. Finally, we report computational results for sample instances from a company.
\end{abstract}

Key words : Real-Time System, Embedded System, Response Time, Local Search, Scheduling Table

\section{Introduction}

A real-time system receives information from the outside, processes it through a sequence of tasks and outputs the result to the outside in short time. Such systems are incorporated in various devices or machines such as mobile phones, information appliances and automobiles, and their importance becomes increasingly large ${ }^{(1)-(3),(6)}$. The behavior of a system is determined by task scheduling. One of the typical scheduling methods is the static priority scheduling, which was analyzed by Liu and Layland (5) and used in many applications. In this method, priority and periods are assigned to tasks by a certain rule (e.g., rate monotonic scheduling, deadline monotonic scheduling), and a schedule is determined by executing tasks periodically based on their periods, where a task with the highest priority is chosen if more than one task is to be executed at the same time. This method, however, suffers from limitations from a viewpoint of CPU utilization (i.e., a resulting schedule usually has idle times), and a new scheduling method is necessary to improve the efficiency of real-time systems.

This paper adopts a scheduling method based on a scheduling table. A schedule of a finite time period is called scheduling table and the method schedules tasks by using the same table repeatedly. In our model of real-time systems, the real-time behavior of a system is evaluated by response times defined on paths, where a path is a sequence of tasks. Each path is associated with a specified time called permissible delay. For each path, during any time interval whose length is the permissible delay, the tasks of the path must be executed in the order specified by the path. We call this the time constraint of the path. Our objective is to schedule tasks so that the time constraints are satisfied for all paths. For static priority 
scheduling, Hashimoto and Yagiura ${ }^{(4)}$ proposed an algorithm that searches for priority and periods such that the resulting schedule satisfies the time constraints as much as possible. Murakami, Tomiyama and Takada ${ }^{(7)}$ proposed a method to determine the priority and the period of each task by simulated annealing, where they considered a very general model of real-time systems containing a next-generation communication technology between modules in a system.

In this paper, to evaluate a schedule, we introduce a concept called active path and propose an efficient evaluation method. Moreover, we propose two methods to obtain a scheduling table that has as small violation of the time constraints as possible. One is a simple local search algorithm in which each neighborhood solution is evaluated with the above mentioned evaluation method. Even though the evaluation method is efficient, the computational cost of this local search is high, because the evaluation procedure is called many times. The other is a more sophisticated local search algorithm that avoids frequent calls to the evaluation procedure. This algorithm uses neighborhood operations that preserve all active paths. Such operations are realized by defining precedence relations between tasks and forbidding the search from violating such relations. Because all active paths are preserved, neighborhood solutions can be evaluated efficiently. Finally, we report computational results for sample instances from a company and confirm the effectiveness of our methods.

\section{Model of real-time systems}

In this section, we describe a model of real-time systems and definitions of tasks and paths.

\subsection{Definition of tasks and paths}

Our model has only one processor that executes tasks and more than one task cannot be executed at a time. Let $\mathcal{T}=\{1,2, \ldots, n\}$ be a set of tasks, where $\mathcal{T}$ is composed of nonpreemptive tasks (that cannot be interrupted during its execution). Each task $i \in \mathcal{T}$ has an execution time $c_{i}(>0)$. Let $\mathcal{P}=\{1,2, \ldots, m\}$ be a set of paths, where a path $p \in \mathcal{P}$ is a sequence of tasks. We denote by $q_{p}(h)$ the $h$ th task in path $p$ and by $\lambda_{p}$ the number of tasks in $p$. Each path $p \in \mathcal{P}$ has a permissible delay $\theta_{p}(>0)$. These given parameters determine an instance of our model.

A real-time system dynamically receives various input (e.g., change in temperature, button input from control panel) from the outside. When a piece of input for a path $p$ is given, the data is processed by $q_{p}(1), q_{p}(2), \ldots, q_{p}\left(\lambda_{p}\right)$ in this order, where $q_{p}(h)$ uses the most recent output of $q_{p}(h-1)$ before the execution of $q_{p}(h)$. Whenever a piece of input is given, the system must output the result of path $p$ within the permissible delay $\theta_{p}$.

Figure 1 shows an example of a schedule composed of three tasks A, B and C, and a path (B, C). We also show executions of each task individually in a separate row. When an execution of $q_{p}(h)$ uses the output of an execution of $q_{p}(h-1)$, the corresponding executions are connected by a solid line.

\subsection{Active path}

Here we introduce the concept of active path for a schedule. Let $\pi_{i}^{j}$ be the $j$ th execution of task $i \in \mathcal{T}$, and let $s_{i}^{j}$ and $e_{i}^{j}$ be the start and finish times of $\pi_{i}^{j}$. We consider the moment when a piece of input data is processed by a path $p$, and let $\left(\pi_{q_{p}(1)}^{j_{1}}, \pi_{q_{p}(2)}^{j_{2}}, \ldots, \pi_{q_{p}\left(\lambda_{p}\right)}^{j_{\lambda_{p}}}\right)$ be the executions of tasks that process this piece of data. The execution of the last task $\pi_{q_{p}\left(\lambda_{p}\right)}^{j_{\lambda_{p}}}$ outputs the result to the outside. Because the execution of task $q_{p}(h+1)$ uses the output of $q_{p}(h)$,

$$
e_{q_{p}(h)}^{j_{h}} \leq s_{q_{p}(h+1)}^{j_{h+1}}
$$

and

$$
j_{h}=\max \left\{k \mid e_{q_{p}(h)}^{k} \leq s_{q_{p}(h+1)}^{j_{h+1}}\right\}
$$




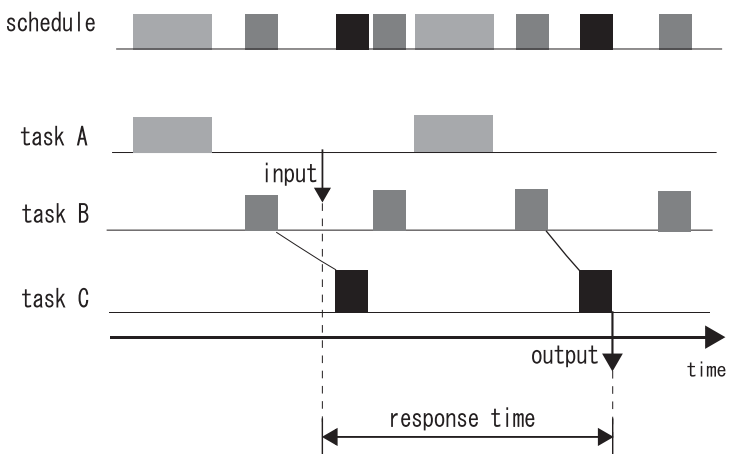

Fig. 1 An example of a schedule

hold for all $h=1,2, \ldots, \lambda_{p}-1$. Furthermore, if $\pi_{q_{p}\left(\lambda_{p}\right)}^{j_{\lambda_{p}}}$ is the first execution of $q_{p}\left(\lambda_{p}\right)$ among those that process the output of $\pi_{q_{p}(1)}^{j_{1}}$, then $\left(\pi_{q_{p}(1)}^{j_{1}}, \pi_{q_{p}(2)}^{j_{2}}, \ldots, \pi_{q_{p}\left(\lambda_{p}\right)}^{j_{\lambda_{p}}}\right)$ is called an active path of $p$. In a schedule, when all the active paths of $p$ are sorted in increasing order of the finish times of the executions of the last task $q_{p}\left(\lambda_{p}\right)$, the $l$ th active path from ahead is called the $l$ th active path of $p$. Let $\phi_{l}^{p}$ be the $l$ th active path of $p$ and let $\phi_{l}^{p}(h)$ be the $h$ th task in this active path, i.e., if $\phi_{l}^{p}=\left(\pi_{q_{p}(1)}^{j_{1}}, \ldots, \pi_{q_{p}\left(\lambda_{p}\right)}^{j_{\lambda_{p}}}\right)$, then $\phi_{l}^{p}(h)=\pi_{q_{p}(h)}^{j_{h}}$.

Figure 2 shows an example of an active path. When an execution of a task uses the output of an execution of another task, the two executions are connected by an arrow. The active path is connected by solid arrows. A piece of input for the system before $\pi_{q_{p}(1)}^{2}$ is reflected to the output at the completion time of $\pi_{q_{p}(3)}^{2}$.

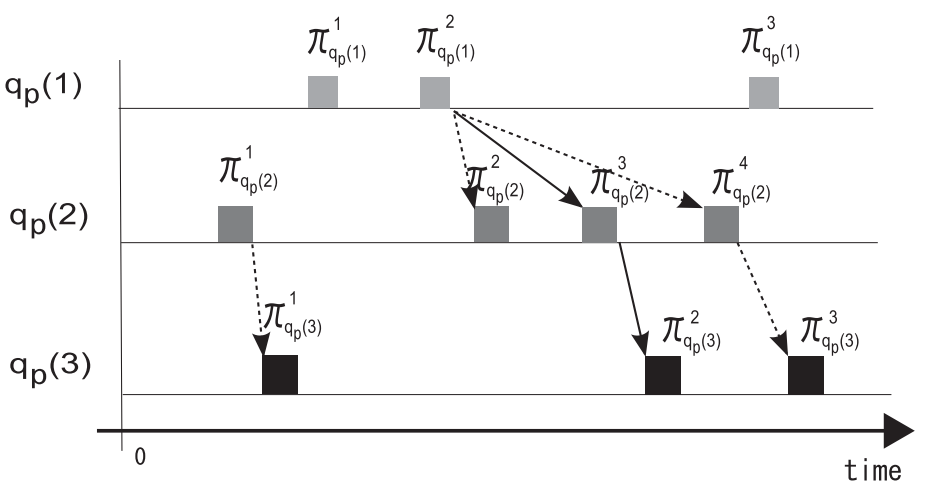

Fig. 2 An example of an active path

Algorithm 1 shows an algorithm of detecting active paths of $p$ for a schedule. Here, $b_{i}$ is the number of executions of task $i$ in the schedule. For each execution of $q_{p}\left(\lambda_{p}\right)$ in the schedule, the algorithm finds all active paths of $p$ by increasing $j_{\lambda_{p}}$ and determining $j_{h}$ that satisfies Equation (2) in the order of $h=\lambda_{p}-1, \ldots, 1$. In Step 6, $s_{q_{p}(h+1)}^{j_{h+1}}$ does not decrease throughout the execution of the algorithm, and hence $j_{h}$ also does not decrease. Hence in Step 6, each execution is referred only once during the whole execution of Algorithm 1, and it takes $\mathrm{O}\left(\sum_{h=1}^{\lambda_{p}} b_{q_{p}(h)}\right)$ time for Step 6. It is easy to see that the time complexity of the other computation is dominated by that of Step 6. In summary, the time complexity of detecting all active paths for $p$ is $\mathrm{O}\left(\Sigma_{h=1}^{\lambda_{p}} b_{q_{p}(h)}\right)$, and all active paths for all $p \in \mathcal{P}$ in the schedule can be detected in $\mathrm{O}\left(\Sigma_{p \in \mathcal{P}} \Sigma_{h=1}^{\lambda_{p}} b_{q_{p}(h)}\right)$ time.

\subsection{Response time}

For a given schedule, we consider the time needed for a piece of input data to be processed by an active path. Let $t_{\text {start }}^{p}(l)$ be the start time of the $l$ th active path of $p$ and $t_{\text {end }}^{p}(l)$ be the finish time $\left(t_{\text {end }}^{p}(0)=0\right.$ is assumed for convenience). Assume that a piece of input 


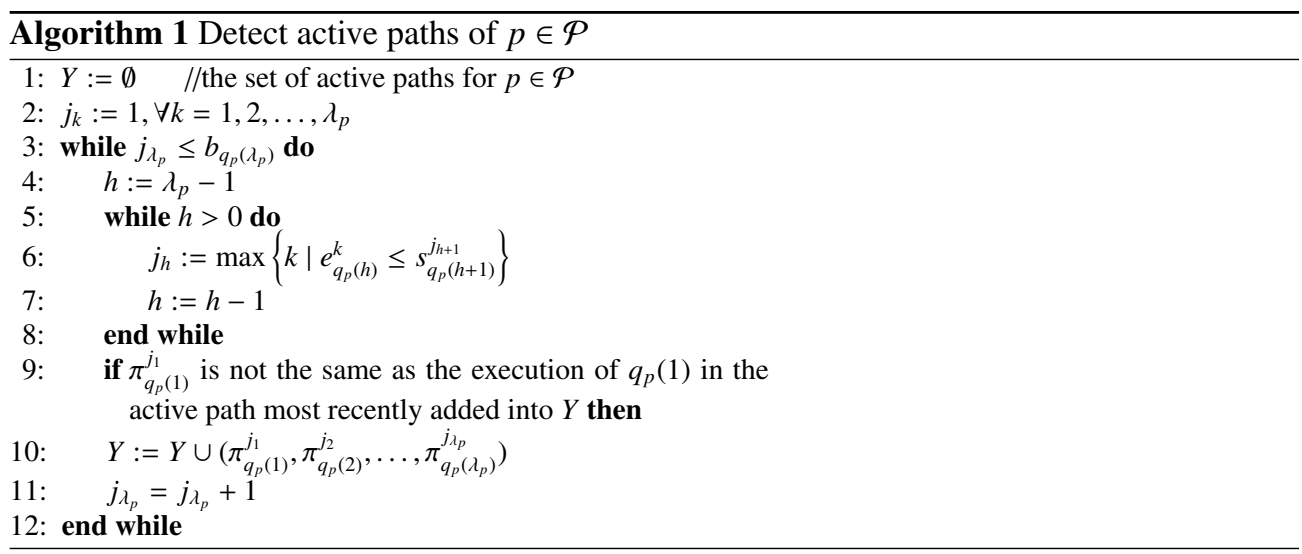

data is given at time $t$ and is processed by the $l$ th active path of $p$. From the assumption, $t_{\text {start }}^{p}(l-1)<t \leq t_{\text {start }}^{p}(l)$ holds and output is obtained at time $t_{\text {end }}^{p}(l)$. The response time is $t_{\text {end }}^{p}(l)-t$, and the worst case is

$$
\lim _{\varepsilon \rightarrow+0}\left\{t_{\text {end }}^{p}(l)-\left(t_{\text {start }}^{p}(l-1)+\varepsilon\right)\right\}=t_{\text {end }}^{p}(l)-t_{\text {start }}^{p}(l-1) .
$$

Let

$$
d_{l}^{p}=t_{\text {end }}^{p}(l)-t_{\text {start }}^{p}(l-1)
$$

and we call $d_{l}^{p}$ the response time of the $l$ th active path of $p$. For a given schedule, let $r_{p}$ be the maximum response time for path $p$ :

$$
r_{p}=\max _{l} d_{l}^{p} .
$$

Then the time constraints for a real-time system can be expressed as

$$
r_{p} \leq \theta_{p}, \quad \forall p \in \mathcal{P} .
$$

Although it is often required that the constraints are satisfied, it is usually difficult to find such a solution. Hence, in this paper, the constraints are allowed to be violated and the violation is minimized. The evaluation criteria for a schedule will be described in Section 2.5.

\subsection{Scheduling table}

In our model, we we assume that a schedule is generated based on a scheduling table. We can schedule tasks by executing them according to a scheduling table and then using the same schedule periodically. Figure 3 shows an example of a schedule generated from a scheduling table. In this figure, tasks are scheduled according to the scheduling table on the top of this figure, where the table is used periodically, e.g., in the time period shown in this figure, the table is repeated three times. For longer time periods, it is repeatedly used until a schedule is made for a required time span.

In general, a scheduling table can include time intervals that have no executed task, i.e., time intervals when the CPU is idle. In this paper, however, we only consider scheduling tables having no such interval, because even if such intervals are removed from a scheduling table with such intervals and shifts the subsequent tasks to the left (i.e., their starting times are made earlier) to remove such intervals, response times will never be increased. For a scheduling table, let $L=\Sigma_{i \in \mathcal{T}} b_{i}$, where $b_{i}$ is the number of executions of task $i$ in the scheduling table. We denote by $\sigma$ the sequence of executions of tasks in the scheduling table, where $\sigma(k)\left(\in\left\{\pi_{i}^{j} \mid\right.\right.$ $\left.\left.j=1,2, \ldots, b_{i}, i \in \mathcal{T}\right\}\right)$ signifies the $k$ th $(k=1,2, \ldots, L)$ execution in the scheduling table.

\subsection{Evaluation of a scheduling table}

This subsection describes how a scheduling table is evaluated. Note that we need to consider response times such that input and the corresponding output cross the border of 


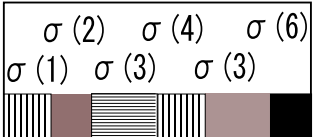

a scheduling table

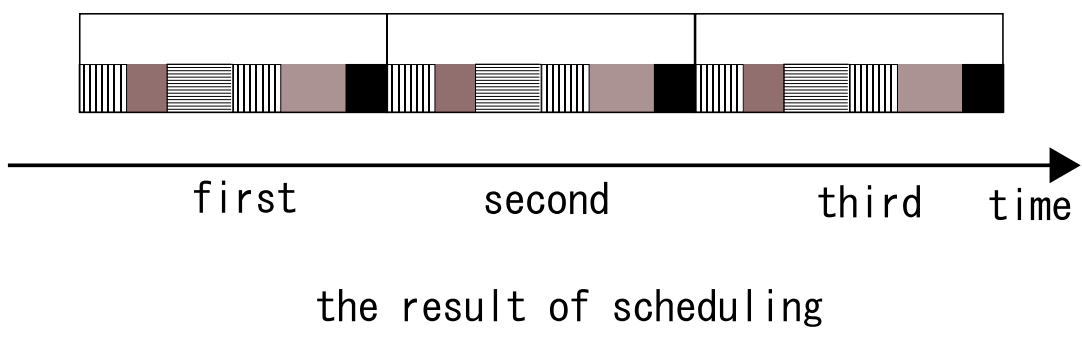

Fig. 3 An example of a schedule defined by a scheduling table

two adjacent rounds (e.g., the first and the second round) of the scheduling table, because a scheduling table is executed periodically. We assume that our algorithms always generate scheduling tables, including those generated during the search, each having at least one active path for each $p \in \mathcal{P}$. Then it is not hard to show that it is sufficient to evaluate the maximum response times of the schedule obtained by repeating the scheduling table twice (i.e., the maximum response time of every path never changes even if the scheduling table is repeated more than twice). To be more precise, for each path $p \in \mathcal{P}$, it suffices to evaluate the maximum response time for the first $T_{p}^{*}$ active paths in the schedule obtained by repeating the table twice, where $T_{p}^{*}$ is defined as follows: the $T_{p}^{*}$ th active path is the first active path such that all executions of tasks are included in the second round. Our algorithm use the following three evaluation criteria for a scheduling table $\sigma$ :

$$
\begin{aligned}
& f_{1}(\sigma)=\max _{p \in \mathcal{P}} \max _{l=2, \ldots, T_{p}^{*}} \frac{\left(d_{l}^{p}-\theta^{p}\right)_{+}}{\theta^{p}} \\
& f_{2}(\sigma)=\sum_{p \in \mathcal{P}} \max _{l=2, \ldots, T_{p}^{*}} \frac{\left(d_{l}^{p}-\theta^{p}\right)_{+}}{\theta^{p}} \\
& f_{3}(\sigma)=\sum_{p \in \mathcal{P}} \sum_{l=2}^{T_{p}^{*}} \frac{\left(d_{l}^{p}-\theta^{p}\right)_{+}}{\theta^{p}},
\end{aligned}
$$

where $(x)_{+}=\max \{x, 0\}$. The first criterion $f_{1}$ means the highest penalty for permissible delay among all response times. The function $f_{2}$ first computes the highest permissible delay of all response times for each path $p$ and then it computes their sum. The third criterion $f_{3}$ means the sum of penalties for permissible delay for all response times. Scheduling tables are compared in the lexicographic order of $\left(f_{1}, f_{2}, f_{3}\right)$, i.e., between two scheduling tables $\sigma$ and $\sigma^{\prime}, \sigma$ is better than $\sigma^{\prime}$ if one of the following three conditions is satisfied: (1) if $f_{1}(\sigma)<f_{1}\left(\sigma^{\prime}\right)$, (2) if $f_{1}(\sigma)=f_{1}\left(\sigma^{\prime}\right)$ and $f_{2}(\sigma)<f_{2}\left(\sigma^{\prime}\right)$, or $(3)$ if $f_{1}(\sigma)=f_{1}\left(\sigma^{\prime}\right), f_{2}(\sigma)=f_{2}\left(\sigma^{\prime}\right)$ and $f_{3}(\sigma)<f_{3}\left(\sigma^{\prime}\right)$. In this paper, we denote $f(\sigma)<f\left(\sigma^{\prime}\right)$ when $\sigma$ is better than $\sigma^{\prime}$.

The criteria $f_{2}$ and $f_{3}$ are introduced for the following reasons. The criterion $f_{1}$ only reflects the response time of an active path (or possibly active paths) which attains the maximum penalty. Hence, if only $f_{1}$ is used, the influence of response times of other active paths are hidden, and there will be many solutions having the same objective value. The criteria $f_{2}$ and $f_{3}$ are introduced to reflect such differences in the objective function. With these criteria, users will get more precise information about schedules than the case where only $f_{1}$ is used. It is also noted that local search algorithms tend to stop in a short time without conducting sufficient search if a max criterion such as $f_{1}$ is used, and better performance is often attained by introducing tie breaking criteria such as $f_{2}$ and $f_{3}$. 


\section{Proposed algorithms}

In this section, we describe two local search algorithms to search for a scheduling table $\sigma$. A local search starts from an initial solution $\sigma$ and repeats replacing $\sigma$ with a better solution in its neighborhood $N(\sigma)$ until no better solution is found in $N(\sigma)$, where $N(\sigma)$ is the set of solutions obtainable from $\sigma$ by applying slight modifications.

\subsection{Initial solution}

To obtain an initial scheduling table, our algorithm simulates a static priority scheduling using the priority and periods of tasks obtained by the algorithm in Ref. (4). The simulation is continued until at least two active paths are formed for every path.

Then the algorithm deletes from the schedule all executions of tasks that are not contained in any active path. Even if it deletes such executions of tasks, response times do not increase. The algorithm then removes all intervals having no executed tasks and shifts the subsequent tasks to the left. The resulting scheduling table is used as an initial solution $\sigma_{\text {init }}$.

\subsection{Local search with shift neighborhood}

Our first local search uses a shift neighborhood $N_{\text {shift }}$, which is the set of solutions obtainable by shift operations. A shift operation moves an execution of a task to another position between two adjacent executions of tasks. For example, from a solution $\sigma=\left(\pi_{2}^{1}, \pi_{1}^{1}, \pi_{3}^{1}, \pi_{4}^{1}, \pi_{1}^{2}\right)$, we can obtain $\sigma^{\prime}=\left(\pi_{1}^{1}, \pi_{3}^{1}, \pi_{2}^{1}, \pi_{4}^{1}, \pi_{1}^{2}\right)$ by shifting $\pi_{2}^{1}$ to the position between $\pi_{3}^{1}$ and $\pi_{4}^{1}$. The size of the shift neighborhood is $\mathrm{O}\left(L^{2}\right)$. In this local search algorithm, whenever a neighborhood solution is generated, it is evaluated by calling the detection algorithm of active paths (Algorithm 1) and then calculating the response times.

\subsection{Local search with fast shift neighborhood}

We consider precedence relations between executions of tasks such that all active paths are preserved after a neighborhood operation if precedence relations are preserved after the operation. This restriction shortens the time to evaluate a neighborhood solution because we do not need to detect active paths for each neighborhood operation. Hence neighborhood solutions can be evaluated efficiently.

3.3.1. Precedence relation For a schedule obtained by applying a scheduling table once, we consider a directed graph $G=(V, E)$, where vertices are corresponding to executions of tasks and edges are corresponding to precedence relations between executions of tasks. To be more precise, the vertex set $V$ is the set of all executions of tasks, and the edge set $E$ is defined as follows:

$$
\begin{aligned}
E= & \left\{\bigcup_{p \in \mathcal{P}} \bigcup_{l=1, \ldots, T_{p}-1} \bigcup_{h=2, \ldots, \lambda_{p}}\left\{\left(\phi_{l}^{p}(h), \phi_{l+1}^{p}(h-1)\right)\right\}\right\} \\
& \cup\left\{\bigcup_{p \in \mathcal{P}} \bigcup_{l=1, \ldots, T_{p}} \bigcup_{h=1, \ldots, \lambda_{p}-1}\left\{\left(\phi_{l}^{p}(h), \phi_{l}^{p}(h+1)\right)\right\}\right\},
\end{aligned}
$$

where $T_{p}$ is the number of all active paths in the schedule. Note that it is complicated to search for a scheduling table considering active paths that bridge two adjacent rounds, and we do not consider such active paths. Figure 4 shows an example of a schedule and the corresponding graph $G$. In the figure, a path $(\mathrm{A}, \mathrm{B}, \mathrm{C})$ is considered. Observe that all edges are directed from left to right in the schedule.

3.3.2. Neighborhood based on precedence relation $\quad$ For two adjacent active paths $\phi_{l-1}^{p}$ and $\phi_{l}^{p}\left(l=2, \ldots, T_{p}\right)$, let $k_{1}$ and $k_{2}$ be the indices that satisfy $\sigma\left(k_{1}\right)=\phi_{l-1}^{p}(1)$ and $\sigma\left(k_{2}\right)=$ $\phi_{l}^{p}\left(\lambda_{p}\right)$, and define a partial sequence $\left(\sigma_{p, l}(1), \sigma_{p, l}(2), \ldots, \sigma_{p, l}\left(k_{2}-k_{1}+1\right)\right)=\left(\sigma\left(k_{1}\right), \sigma\left(k_{1}+\right.\right.$ $\left.1), \ldots, \sigma\left(k_{1}+k_{2}-k_{1}\right)\right)$. We consider a shift operation that moves an execution in $\sigma_{p, l}$ to the left of $\sigma\left(k_{1}\right)$ or to the right of $\sigma\left(k_{2}\right)$ without violating the precedence relations (i.e., without reversing the direction of any edge). By such an operation, an execution of a task in $\sigma_{p, l}$ is 


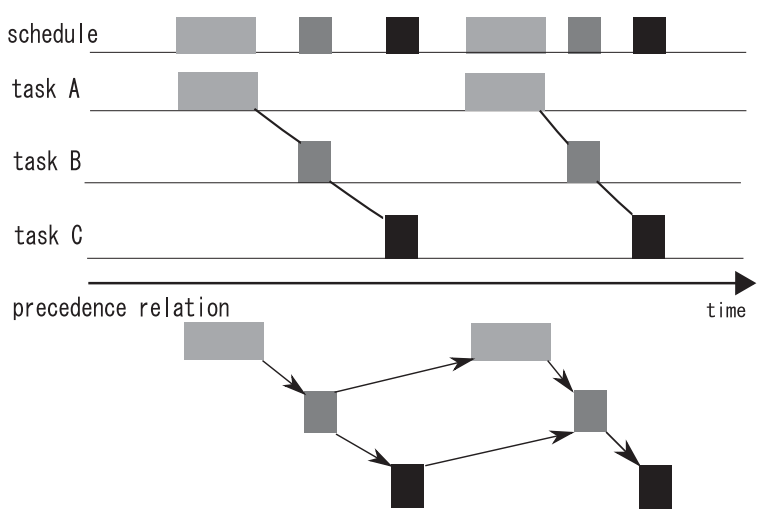

Fig. 4 An example of precedence relations
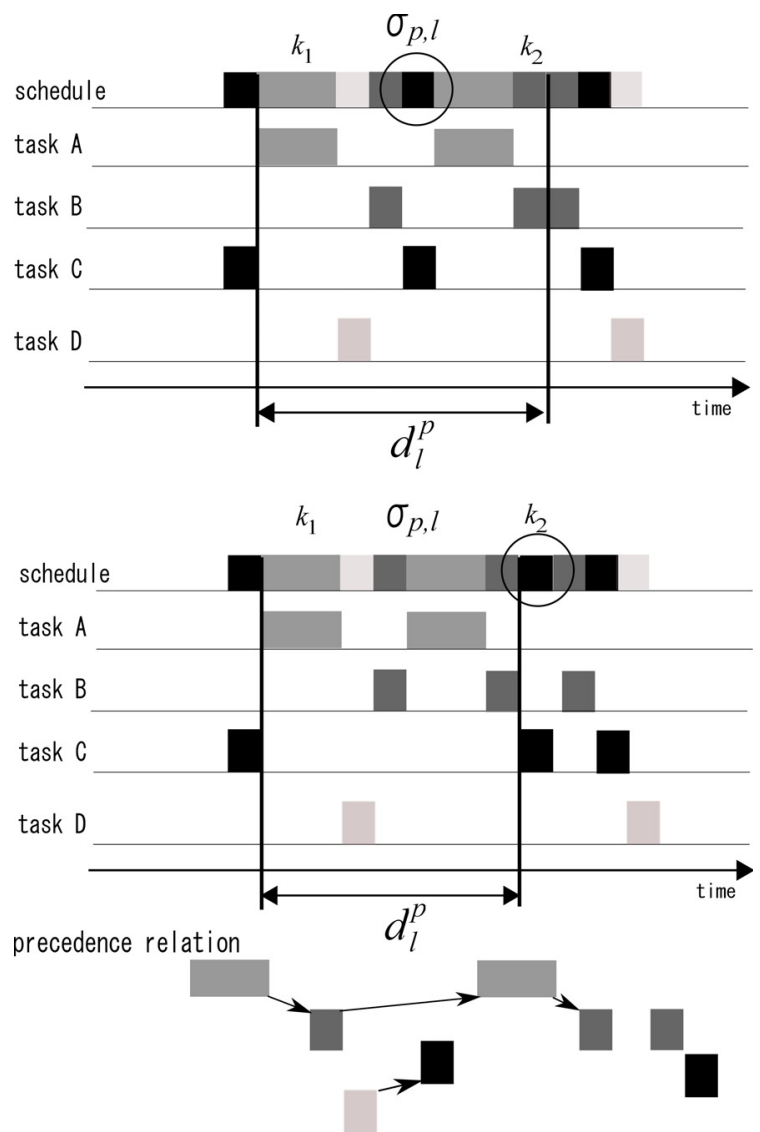

Fig. 5 An example of improving by shifting a task

shifted to the outside of $\sigma_{p, l}$, and $d_{l}^{p}$ is decreased by the execution time of the task. Figure 5 shows an example of shifting an execution to the outside of an interval $\sigma_{p, l}$. The response time $d_{l}^{p}$ is improved and all precedence relations are preserved. If all active paths are preserved after a shift operation, the neighborhood solution can be evaluated efficiently by calculating only those changes of response times caused by the operation without detecting active paths. However, it is not trivial to find all executions in $\sigma_{p, l}$ that preserve all active paths after such shift operations.

For each combination of $p$ and $l$, we restrict the candidates of shift moves to the leftmost (resp., rightmost) execution among those that do not violate precedence conditions when a shift move to the left (resp., right) is considered. The leftmost candidate can be found by first letting $\sigma_{p, l}(1)$ marked and all elements $\sigma_{p, l}(k)\left(k=2,3, \ldots, k_{2}-k_{1}+1\right)$ unmarked, and then repeating the following for $k=1,2, \ldots$ until a candidate is found: if $\sigma_{p, l}(k)$ is unmarked, then stop (this is the execution we would like to find); otherwise, let $u$ be the 
vertex corresponding to $\sigma_{p, l}(k)$, and mark every element $\sigma_{p, l}\left(k^{\prime}\right)$ that corresponds to vertex $v$ having an edge $(u, v) \in E$ from $u$. The rightmost candidate can be found similarly. Let $S(\sigma, p, l, \mathrm{~L})$ (resp., $S(\sigma, p, l, \mathrm{R}))$ be the scheduling table that is obtained by shifting such a task to the left (resp., right) end of interval $\sigma_{p, l}$ for $l=2, \ldots, T_{p}$. Furthermore, to consider both ends of the scheduling table, we define two intervals $\sigma_{p, 1}$ and $\sigma_{p, T_{p}+1}$, where $\sigma_{p, 1}$ consists of executions from $\sigma(1)$ to $\phi_{1}^{p}\left(\lambda_{p}\right)$, and $\sigma_{p, T_{p}+1}$ consists of executions from $\phi_{T_{p}}^{p}(1)$ to $\sigma(L)$. Then we consider a shift operation to move an execution in $\sigma_{p, 1}$ (resp., $\sigma_{p, T_{p}+1}$ ) to its right end (resp., left end) in a similar way. The solution obtained by such an operation is denoted by $S(\sigma, p, 1, \mathrm{R})$ (resp., $\left.S\left(\sigma, p, T_{p}+1, \mathrm{~L}\right)\right)$.

The search order of the local search with the fast shift neighborhood is as follows. First, the algorithm searches for an improved neighborhood solution $S(\sigma, p, l, \mathrm{R})$ until there is no such solution with for all $p$ and $l$. Then the algorithm searches for an improved solution $S(\sigma, p, l, \mathrm{~L})$ similarly, and when there is no improvement with $S(\sigma, p, l, \mathrm{~L})$, it returns to the search for an improved $S(\sigma, p, l, \mathrm{R})$. These two types of neighborhoods are thus searched alternately, and when no improvement is found with both $S(\sigma, p, l, \mathrm{~L})$ and $S(\sigma, p, l, \mathrm{R})$, the algorithm outputs the current scheduling table and halts.

\section{Computational experiments}

To confirm the performance of our algorithms, we conducted computational experiments for sample instances provided from a company. The algorithms were coded in the $\mathrm{C}$ language and run on a PC (Core2Duo CPU 2.4GHz, 2GB memory). Although the original instances have been generated in consideration of real applications, it was easy to find scheduling tables that satisfy the time constraints. Hence, we modified them by shortening all permissible delays by multiplying an identical factor. This corresponds to the case where we are required to use a slower CPU.

Table 1 and 2 show the computational results of the local search algorithm with the shift neighborhood and the local search algorithm with the fast shift neighborhood, respectively. In these tables, initial solutions are denoted by $\sigma_{\text {init }}$ and output solutions are denoted by $\sigma_{\text {out }}$. Column "time" shows the computation time in seconds when the algorithm stopped at a locally optimal solution except for instances "tw-200-2942" and "tw-1000-45314." We stopped our algorithm when the computation time exceeded 50,000 seconds for the instance "tw-2002942" and "tw-1000-45314," and in these cases, $\sigma_{\text {out }}$ is the incumbent solution at the time it was stopped. From the results of Table 1 and 2, we can observe that the quality of the local

Table 1 Computational results for the local search with the shift neighborhood

\begin{tabular}{lrrrrrrrrr}
\hline instance & $|\mathcal{T}|$ & $|\mathcal{P}|$ & $f_{1}\left(\sigma_{\text {init }}\right)$ & $f_{2}\left(\sigma_{\text {init }}\right)$ & $f_{3}\left(\sigma_{\text {init }}\right)$ & $f_{1}\left(\sigma_{\text {out }}\right)$ & $f_{2}\left(\sigma_{\text {out }}\right)$ & $f_{3}\left(\sigma_{\text {out }}\right)$ & time \\
\hline tw-8-4 & 8 & 4 & 63.00 & 173 & 2125 & 23.00 & 55 & 663 & 328.02 \\
tw-18-10 & 18 & 10 & 244.44 & 1807 & 12,657 & 113.89 & 857 & 5662 & 403.39 \\
tw-23-13 & 23 & 13 & 319.57 & 2446 & 11,383 & 132.61 & 1124 & 5183 & 586.89 \\
tw-200-2942 & 200 & 2942 & 27.20 & 5470 & 6394 & 21.50 & 8258 & 10327 & $>50,000$ \\
tw-1000-45314 & 1000 & 45,314 & 51.24 & 268,107 & 355,343 & 51.20 & 269,535 & 356,829 & $>50,000$ \\
\hline
\end{tabular}

search with the fast shift neighborhood is worse than that of the local search with the shift neighborhood for small instances "tw-8-4," "tw-18-10" and "tw-23-13" with up to 23 tasks and 13 paths, but the opposite holds for much larger instances "tw-200-2942" and "tw-100045314" with up to 1000 tasks and 45,314 paths. Moreover, the computation time of the local search with the fast shift neighborhood is much shorter.

Next, we show how the value of $f_{1}$ changes during the execution of the algorithms. Figures from 6 to 10 show the value of $f_{1}$ against time for each instance. In the figures, the vertical (resp., horizontal) axis denotes the value of $f_{1}$ (resp., computation time, where the scale is logarithmic) and "shift" (resp., "fast shift") represents the result of the local search with the shift neighborhood (resp., the local search with the fast shift neighborhood). We can observe that the local search algorithm with the fast shift neighborhood improves a solution 
Table 2 Computational result for the local search with the fast shift neighborhood

\begin{tabular}{lrrrrrrrrr}
\hline instance & $|\mathcal{T}|$ & $|\mathcal{P}|$ & $f_{1}\left(\sigma_{\text {init }}\right)$ & $f_{2}\left(\sigma_{\text {init }}\right)$ & $f_{3}\left(\sigma_{\text {init }}\right)$ & $f_{1}\left(\sigma_{\text {out }}\right)$ & $f_{2}\left(\sigma_{\text {out }}\right)$ & $f_{3}\left(\sigma_{\text {out }}\right)$ & time \\
\hline tw-8-4 & 8 & 4 & 63.00 & 173 & 2125 & 57.00 & 137 & 1617 & 0.03 \\
tw-18-10 & 18 & 10 & 244.44 & 1807 & 12,657 & 182.78 & 1166 & 7483 & 0.03 \\
tw-23-13 & 23 & 13 & 319.57 & 2446 & 11,383 & 259.13 & 1879 & 7654 & 0.03 \\
tw-200-2942 & 200 & 2942 & 27.20 & 5470 & 6394 & 12.20 & 755 & 759 & 2.88 \\
tw-1000-45314 & 1000 & 45,314 & 51.24 & 268,107 & 355,343 & 44.15 & 60,010 & 66,984 & 3995 \\
\hline
\end{tabular}

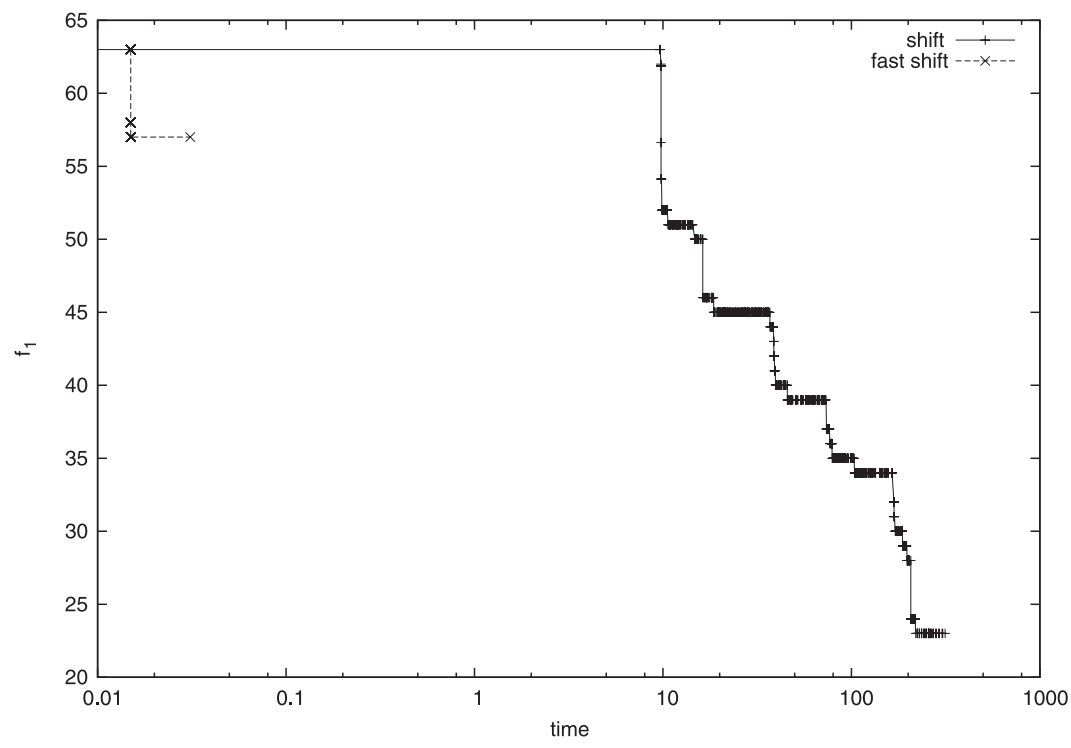

Fig. 6 The value of $f_{1}$ of the two algorithms for tw-8-4 against time

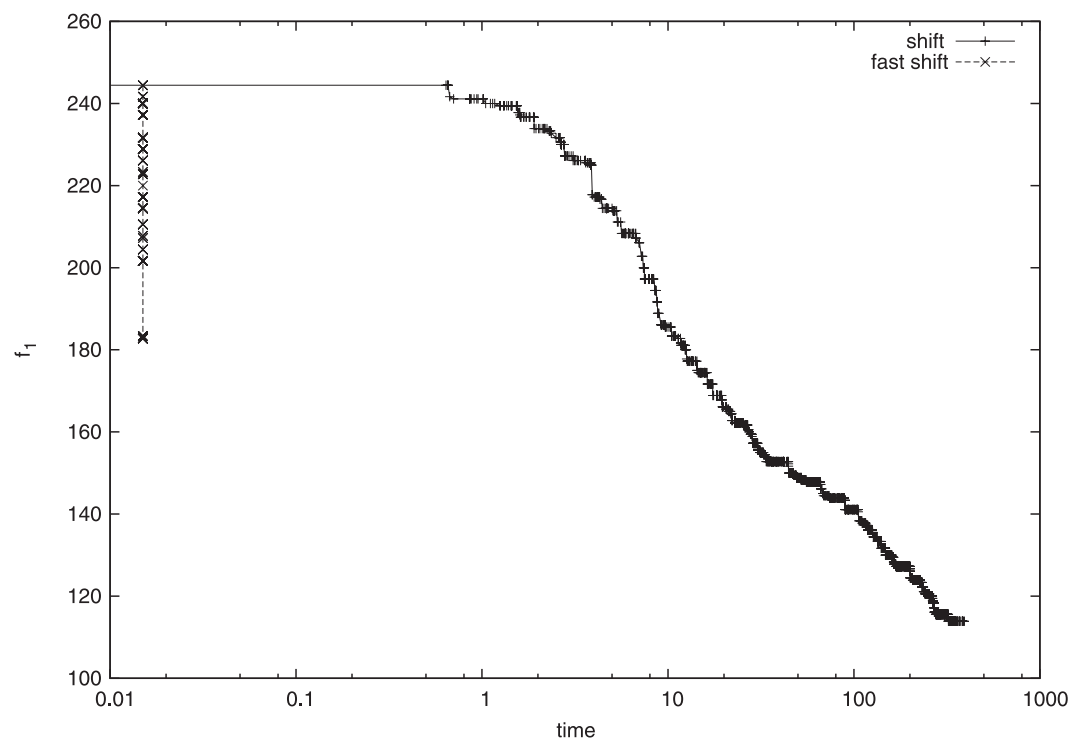

Fig. 7 The value of $f_{1}$ of the two algorithms for tw-18-10 against time

much faster than the other. If a better solution for a large instance is needed, the local search with the shift neighborhood is impractical and metaheuristics with the local search with the fast shift neighborhood would be reasonable.

\section{Conclusion}

In our model of real-time systems, the real-time behavior of a system is evaluated by response times defined on paths, where a path is a sequence of tasks. We adopted a scheduling method based on a scheduling table, which specifies a schedule of a finite time period and is used repeatedly to define the schedule of longer time periods. To evaluate a scheduling table, 


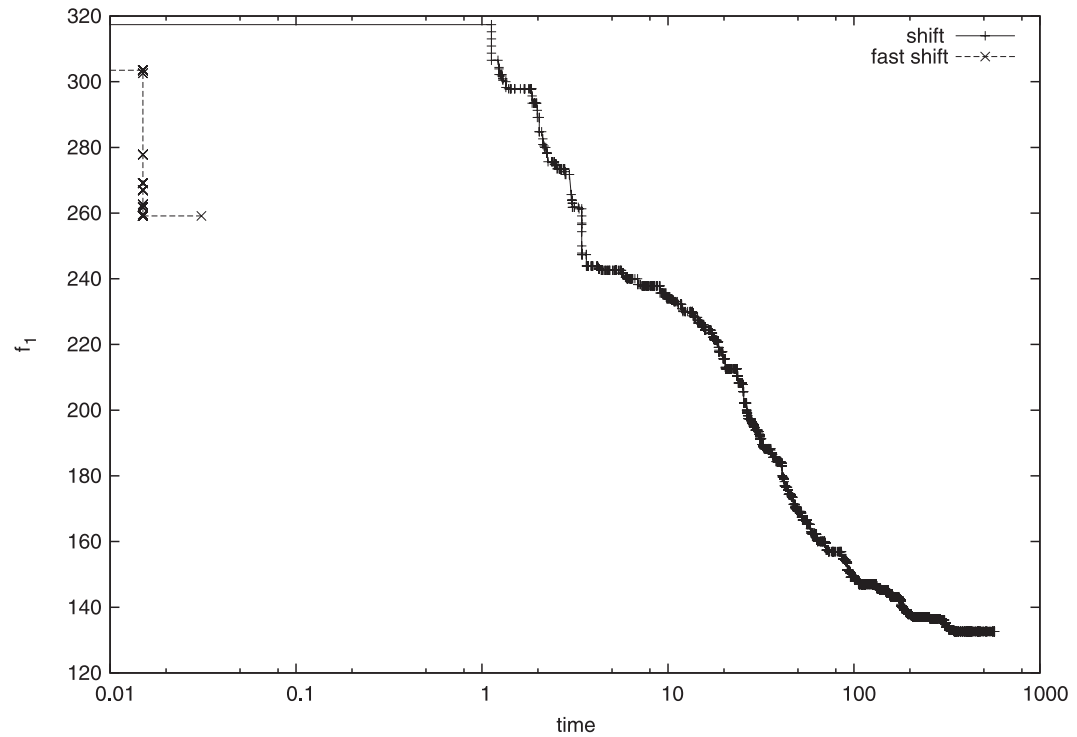

Fig. 8 The value of $f_{1}$ of the two algorithms for tw-23-13 against time

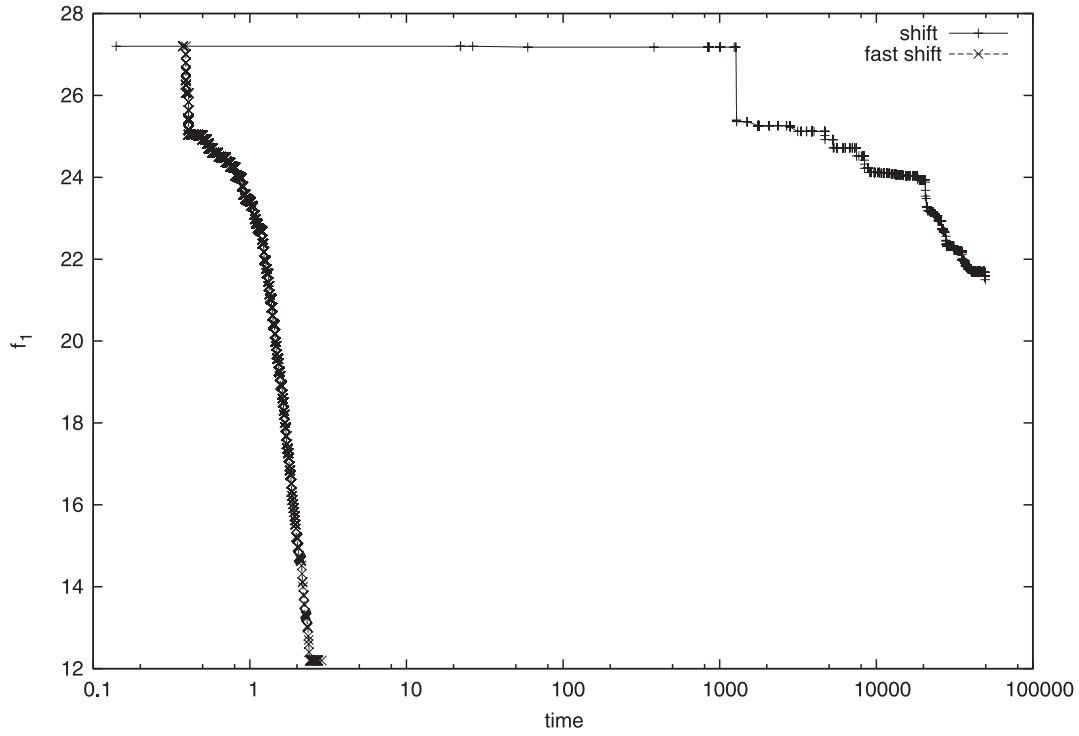

Fig. 9 The value of $f_{1}$ of the two algorithms for tw-200-2942 against time

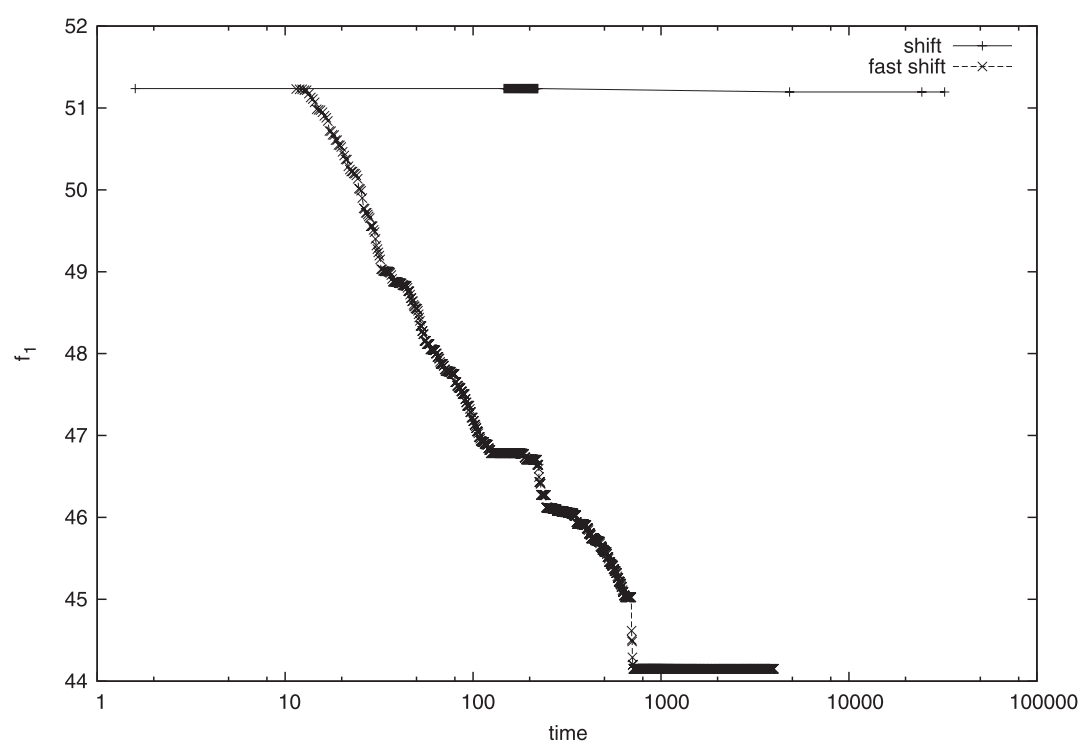

Fig. 10 The value of $f_{1}$ of the two algorithms for tw-1000-45314 against time 
we introduced a concept called active path and proposed an efficient evaluation method. We then proposed two local search algorithms to find a scheduling table. The local search with the standard shift neighborhood invokes the evaluation method whenever a neighborhood solution is generated. The other local search with the fast shift neighborhood avoids frequent calls to the evaluation method by preserving all active paths. From computational results, it was confirmed that the local search with the fast shift neighborhood succeeded in shortening the computation time greatly and obtained better solutions for large instances in shorter time.

\section{References}

( 1 ) Baruah, S. and Goossens, J., Scheduling real-time tasks: Algorithms and complexity, In Leung, J. Y.-T., editor, Handbook of Scheduling: Algorithms, Models, and Performance Analysis, chapter 28. Chapman Hall/CRC Press, (2004).

( 2 ) Błażewicz, J., Ecker, K. H., Pesch, E., Schmidt, G., and Węglarz, J., Scheduling in Hard Real-Time Systems, (2007), pp.243-269, Springer.

( 3 ) Buttazzo, G. C., Hard real-time computing systems: predictable scheduling algorithms and applications, (2004), Springer, 2 edition.

( 4 ) Hashimoto, H. and Yagiura, M., An LP-based algorithm for scheduling preemptive and/or non-preemptive real-time tasks, Journal of Advanced Mechanical Design, Systems, and Manufacturing, Vol.4, (2010), pp.578-587.

( 5 ) Liu, C. L. and Layland, J. W., Scheduling algorithms for multiprogramming in a hard real-time environment, Journal of the ACM, Vol.20, (1973), pp.46-61.

( 6 ) Liu, J. W. S., Real-Time Systems, (2000), Prentice Hall.

( 7 ) Murakami, N., Tomiyama, H., and Takada, H., A static scheduling method for distributed automotive control systems (real time processing) (in Japanese), Information Processing Society of Japan (IPSJ), Vol.48, (2007), pp.203-215. 